\title{
OBSTACLE PROBLEMS FOR SCALAR CONSERVATION LAWS
}

\author{
LAURENT LEVI ${ }^{1}$
}

\begin{abstract}
In this paper we are interested in bilateral obstacle problems for quasilinear scalar conservation laws associated with Dirichlet boundary conditions. Firstly, we provide a suitable entropy formulation which ensures uniqueness. Then, we justify the existence of a solution through the method of penalization and by referring to the notion of entropy process solution due to specific properties of bounded sequences in $L^{\infty}$. Lastly, we study the behaviour of this solution and its stability properties with respect to the associated obstacle functions.
\end{abstract}

Résumé. Ce travail a pour objet l'étude de problèmes d'obstacles bilatéraux pour des lois de conservation scalaires quasi-linénaires du premier ordre associées à des conditions aux limites de Dirichlet. On donne d'abord une formulation entropique qui garantit l'unicité. On justifie alors l'existence d'une solution par utilisation de la méthode de pénalisation et au moyen de la notion de processus entropique solution due aux propriétés des suites bornées dans $L^{\infty}$. Enfin, on étudie le comportement de cette solution et ses propriétés de stabilité en fonction des contraintes d'obstacle associées.

Mathematics Subject Classification. 35L65, 35R35, 35L85.

Received: March, 2000. Revised: February 7, 2001.

\section{INTRODUCTION AND MATHEMATICAL FRAMEWORK}

\subsection{Physical motivations}

Obstacle problems for first-order hyperbolic operators have been introduced by Bensoussan and Lions [4] in 1973, as part of the study of cost-functions associated with deterministic processes. In this paper we are interested in non-homogeneous Dirichlet problems for general scalar conservation laws associated with a forced bilateral constraint. The physical motivations of such a study are diverse: for example, in the hydrological field we consider the simplified modelling of one phase saturating the subsoil and made of two components without any chemical interactions: water and the component $c$. Depending on the geological nature of the subsoil, the molecular diffusion-dispersion effects can be neglected in favor of the transport ones. Hence, by referring to [5], given the distribution of temperatures $T$ and the pressure field $P$ of the fluid phase, the transcription of the mass conservation law for $c$ provides the equality ruling the mass fraction $\omega_{c}$ :

$$
\partial_{t} \omega_{c}-\frac{k(x)}{\mu\left(\omega_{c}\right)} \nabla \omega_{c}\left(\nabla P-\rho\left(T, \omega_{c}\right) \vec{g}\right)=0
$$

\footnotetext{
Keywords and phrases. Obstacle problem, conservation laws, entropy solution.

${ }^{1}$ University of Pau, CNRS, Laboratory of Applied Mathematics ERS 2055, I.P.R.A., Avenue de l'Université, 64000 Pau, France. e-mail: laurent.levi@univ-pau.fr
} 
where $k(x)$ denotes the absolute permeability at the point $x, \mu$ is the dynamic viscosity of the fluid phase and $\rho\left(T, \omega_{c}\right)$ its voluminal mass, defined by the composition $\omega_{c}$ at the temperature $T$. Lastly, $\vec{g}$ is the gravity acceleration vector. Furthermore, $\omega_{c}$ must satisfy the bilateral obstacle condition:

$$
\theta_{1, c}(T, P) \leq \omega_{c} \leq \theta_{2, c}(T, P),
$$

where $\theta_{1, c}(T, P)$ and $\theta_{2, c}(T, P)$ are saturation thresholds. Beyond these values the appearance of a new phase, liquid or solid, for the same number of components changes the thermodynamical nature of the considered system and the latter cannot be described through a simplified balance equation.

In petroleum engineering one is led to examine the case of a unique oil phase, saturating the reservoir rock, which is made of two components: heavy and light oil. Within the context of isothermal flows, when pressure $P$ is a determined sufficiently smooth function, writing the heavy oil component's mass conservation law leads to the first-order equality:

$$
\partial_{t} C_{o}^{h}-\operatorname{Div}\left[k(P) \frac{C_{o}^{h}}{\mu_{o}\left(C_{o}^{h}\right)}\left(\vec{\nabla} P-\rho_{o}\left(C_{o}^{h}\right) \vec{g}\right)\right]=0 .
$$

Moreover, $C_{o}^{h}$ has to fulfill the unilateral obstacle condition:

$$
\theta(P) \leq C_{o}^{h},
$$

at pressure $P$. Indeed, beyond this value the appearance of a gaseous phase for the same number of components shows that the molar fraction of the pseudo heavy constituent is determined by the knowledge of the pressure; thus, the proposed model considers the dissolution of gas into oil, yet it excludes the gas-liberating phenomenon. A comprehensive treatment of those problems may be found in Gagneux and Madaune-Tort's book [10], particularly the Black-Oil model that we have referred to.

\subsection{Mathematical setting and assumptions on data}

The previous motivations lead us to introduce the first-order quasilinear hyperbolic operator including a reaction term

$$
\mathbb{H}(t, x, .): u \rightarrow \partial_{t} u+\sum_{i=1}^{p} \partial_{x_{i}} f_{i}(t, x, u)+g(t, x, u),
$$

and two measurable obstacle-functions $\theta_{1}$ and $\theta_{2}$ such that

$$
\left.\theta_{1} \leq \theta_{2} \text { a.e. on } Q=\right] 0, T[\times \Omega,
$$

where $T$ is a strictly positive and finite real and $\Omega$ is a subdomain of $\mathbb{R}^{p}, p \geq 1$, with a Lipschitz boundary $\Gamma=\partial \Omega$. Then, the purpose of this article is to study the free boundary problem:

$$
\begin{gathered}
\theta_{1} \leq u \leq \theta_{2} \text { a.e. on } Q \\
\left(u-\theta_{1}\right) \mathbb{H}(t, x, u) \leq 0,\left(u-\theta_{2}\right) \mathbb{H}(t, x, u) \leq 0,\left(u-\theta_{1}\right)\left(u-\theta_{2}\right) \mathbb{H}(t, x, u)=0 \text { on } Q, \\
\left.u=u_{\Gamma} \text { on (a part of }\right) \Sigma, u(0, .)=u_{0} \text { on } \Omega
\end{gathered}
$$

where $u_{0}$ and $u_{\Gamma}$ are two measurable and bounded functions respectively on $\Omega$ and $\left.\Sigma=\right] 0, T[\times \Gamma$ and such that:

$$
\begin{aligned}
\theta_{1}(0, .) & \leq u_{0} \leq \theta_{2}(0, .) \text { a.e. on } \Omega, \\
\theta_{1} & \leq u_{\Gamma} \leq \theta_{2} \text { a.e. on } \Sigma .
\end{aligned}
$$


What is more, in the rest of this paper we assume that the data satisfy the following assumptions:

- $f_{i}, i \in\{1, . ., p\}$, (respect. $g$ ) is a $\mathcal{C}^{1}$-class function on $\bar{Q} \times \mathbb{R}$ (respect. a continuous function on $\bar{Q} \times \mathbb{R}$ ). In addition, $\partial_{x_{i}} f_{i}, i \in\{1, . ., p\}$, and $g$ are Lipschitzian with respect to the third variable, uniformly in $(t, x)$. - $\theta_{1}$ and $\theta_{2}$ are elements of $W^{1,+\infty}(Q)$.

Those hypotheses guarantee the existence of the quantity $M(t)$ defined for any real $t$ of $[0, T]$ by

$$
M(t)=\max \left(\left\|u_{0}\right\|_{L^{\infty}(\Omega)} ;\left\|u_{\Gamma}\right\|_{L^{\infty}(\Sigma)} ;\left\|\theta_{1}\right\|_{L^{\infty}(Q)}\right) \mathrm{e}^{C_{1} t}+\frac{\mathrm{e}^{C_{1} t}-1}{C_{1}} \max _{[0, T] \times \bar{\Omega}}|\mathbb{H}(t, x, 0)|,
$$

where $C_{1}$ is the sum of the Lipschitz constants with respect to $u$ of $g$ and $\partial_{x_{i}} f_{i}, i \in\{1, . ., p\}$.

- $\boldsymbol{\nu}$ denotes the outer normal vector defined a.e. on $\Gamma$.

We first have to provide a mathematical formulation for obstacle problem (1), (2) and (3) by keeping in mind that on the one hand, for a general first-order quasilinear equation, it is classical to refer to an entropy criterium which warrants uniqueness. On the other hand, without any assumptions on the sign of the source term for $\mathbb{H}$, the introduction of obstacle constraints on the initial datum does not a priori pass on to the solution. Therefore we need an entropy formulation taking these constraints into account. Lastly, since $u_{0}$ and $u_{\Gamma}$ are only bounded functions, we need to refer to the works of Otto in [14], Chapter 2, which introduce a new formulation of boundary conditions for quasilinear hyperbolic equations, since in the case of $L^{\infty}(Q)$-solutions we cannot use the notion of a trace on $\Gamma$, available for a function of bounded variation on $Q$. With this view, in the rest of this paper, we denote $\mathbf{f}=\left(f_{1}, f_{2}, . ., f_{p}\right)$ and

$$
\begin{aligned}
\mathbf{F}(u, v) & =\operatorname{sgn}(u-v)[\mathbf{f}(t, x, u)-\mathbf{f}(t, x, v)], \\
G(u, v) & =\operatorname{sgn}(u-v)\left[\boldsymbol{\nabla} \cdot \mathbf{f}(t, x, v)+g(t, x, u)+\partial_{t} v\right], \\
\mathcal{L}(u, v, w) & =|u-v| \partial_{t} w+\mathbf{F}(u, v) \cdot \boldsymbol{\nabla} w-G(u, v) w,
\end{aligned}
$$

the dependence on time and space variables of the non-linearities $\mathbf{F}$ and $G$ not being essential to comprehension. We thus say by denoting for any real $k$

$$
K(t, x)=k\left(\theta_{2}(t, x)-\theta_{1}(t, x)\right)+\theta_{1}(t, x) .
$$

Definition 1.1. A measurable function $u$ is called an entropy solution to (1), (2), (3) if it satisfies:

i) the bilateral constraint

$$
\theta_{1}(t, x) \leq u(t, x) \leq \theta_{2}(t, x) \text { for a.e. }(t, x) \text { in } Q,
$$

ii) the entropy condition, for all functions $\xi$ of $\mathcal{D}_{+}(] 0, T[\times \Omega)$ for any real $k$ of $[0,1]$,

$$
\int_{Q} \mathcal{L}(u, K, \xi) \mathrm{d} x \mathrm{~d} t \geq 0 .
$$

iii) the initial condition $u_{0} \in L^{\infty}(\Omega)$ in the $L^{1}$-sense:

$$
\text { ess } \lim _{t \rightarrow 0^{+}} \int_{\Omega}\left|u(t, x)-u_{0}(x)\right| \mathrm{d} x=0,
$$

iv) the boundary condition $u_{\Gamma} \in L^{\infty}(\Sigma)$, in the weak sense:

$$
\text { ess } \lim _{\tau \rightarrow 0^{-}} \int_{\Sigma} \mathcal{F}\left(u(\sigma+\tau \boldsymbol{\nu}), u_{\Gamma}, K\right) \cdot \boldsymbol{\nu} \zeta \mathrm{d} \sigma \geq 0
$$


for any real $k$ of $[0,1]$ and for all functions $\zeta$ of $L_{+}^{1}(\Sigma)$ where, for any real $a, b$ and $c$,

$$
2 \mathcal{F}(a, b, c)=\mathbf{F}(a, b)-\mathbf{F}(c, b)+\mathbf{F}(a, c) .
$$

\section{The Uniqueness PROOF}

Let us verify that the weak entropy formulation proposed in Definition 1.1 ensures the uniqueness of a solution. With this view we refer to Otto's works in [14], Chapter 2, relative to the study of Dirichlet problems for homogeneous scalar conservation laws and we adapt them to the present situation where $\mathbb{H}$ involves a reaction term and $K$ is a time-and-space-depending function. Thus, boundary conditions (8) allow us to provide an $L^{1}$ continuity result with respect to boundary data thanks to the following relation:

Lemma 2.1. Let $u$ be an entropy solution to obstacle problem (1),(2),(3) in the sense of Definition 1.1. Then, for any real $k$ of $[0,1]$ and for any function $\xi$ of $\mathcal{D}_{+}(] 0, T\left[\times \mathbb{R}^{p}\right)$,

$$
-\int_{Q} \mathcal{L}(u, K, \xi) \mathrm{d} x \mathrm{~d} t \leq e s s \lim _{\tau \rightarrow 0^{-}} \int_{\Sigma} \boldsymbol{F}\left(u(\sigma+\tau \boldsymbol{\nu}), u_{\Gamma}\right) \cdot \boldsymbol{\nu} \xi \mathrm{d} \sigma-\int_{\Sigma} \boldsymbol{F}\left(K, u_{\Gamma}\right) \cdot \boldsymbol{\nu} \xi \mathrm{d} \sigma
$$

Proof. By adapting Otto's reasoning, one may be sure that since (6) holds, then for any function $\delta$ of $L_{+}^{1}(\Sigma)$,

$$
\text { ess } \lim _{\tau \rightarrow 0^{-}} \int_{\Sigma} \mathbf{F}\left(u(\sigma+\tau \boldsymbol{\nu}), w\left(\theta_{2}(\sigma+\tau \boldsymbol{\nu})-\theta_{1}(\sigma+\tau \boldsymbol{\nu})\right)+\theta_{1}(\sigma+\tau \boldsymbol{\nu})\right) \cdot \boldsymbol{\nu} \delta \mathrm{d} \sigma \text { exists, }
$$

for any $w$ of $L^{\infty}(\Sigma)$ such that $0 \leq w \leq 1$ a.e. on $\Sigma$. So, due to the smoothness of $\mathbf{f}$ and $\theta_{i}, i=1,2$ we infer:

$$
\text { ess } \lim _{\tau \rightarrow 0^{-}} \int_{\Sigma} \mathbf{F}(u(\sigma+\tau \boldsymbol{\nu}), w) \delta \mathrm{d} \sigma \text { exists, }
$$

for any $w$ of $L^{\infty}(\Sigma)$ such that $\theta_{1} \leq w \leq \theta_{2}$ a.e. on $\Sigma$.

On the other hand, entropy relation (6) ensures that for any function $\zeta$ of $\mathcal{D}(] 0, T\left[\times \mathbb{R}^{p}\right)$,

$$
-\int_{Q} \mathcal{L}(u, K, \xi) \mathrm{d} x \mathrm{~d} t \leq-e s s \lim _{\tau \rightarrow 0^{-}} i n f \int_{\Sigma} \mathbf{F}(u(\sigma+\tau \boldsymbol{\nu}), K(\sigma+\tau \boldsymbol{\nu})) \cdot \boldsymbol{\nu} \xi \mathrm{d} \sigma .
$$

This inequality and boundary condition (8) give (9) thanks to the regularities of $\mathbf{f}$ and $\theta_{i}$.

\subsection{The Kruskov relation}

The proof uses that one developed by Kruskov [12] by splitting the time and space variables into two. However, the introduction of a forced bilateral obstacle condition gives rise to the choice of an entropy family depending on $(t, x)$, through functions $\theta_{1}$ and $\theta_{2}$. Even so, the next $L^{1}$-stability result holds:

Theorem 2.1. Let $u$ and $v$ be two entropy solutions of obstacle problem (1), (2), (3) in the sense of Definition 1.1 and corresponding to boundary conditions $\left(u_{0}, u_{\Gamma}\right)$ and $\left(v_{0}, v_{\Gamma}\right)$. Then, for a.e. $t$ in $] 0, T[$,

$$
\int_{\Omega}|u(t, x)-v(t, x)| \mathrm{d} x \leq\left(A \int_{0}^{t} \int_{\Gamma}\left|u_{\Gamma}-v_{\Gamma}\right| \mathrm{d} \sigma+\int_{\Omega}\left|u_{0}-v_{0}\right| \mathrm{d} x\right) \mathrm{e}^{M_{g}^{\prime} t},
$$

where $A$ and $M_{g}^{\prime}$ are respectively the Lipschitz constants of $\mathbf{f}$ and $g$ with respect to their third variable. 
Proof. Let $m$ be an element of $\mathbb{N}^{*}$ and let $\rho_{m}$ be the standard mollifer sequence in $\mathbb{R}^{m}$. For each $\epsilon>0$, let $\left(\rho_{m, \epsilon}\right)_{\epsilon>0}$ be the sequence defined by:

$$
\rho_{m, \epsilon}(x)=\frac{1}{\epsilon^{m}} \rho_{m}\left(\frac{x}{\epsilon}\right)
$$

Let $p$ and $\tilde{p}$ be elements of $Q$. To simplify the writing we add a "tilde" superscript to any function in "tilde" variables. Thus, we denote $l$ and $k$ the functions of $L^{\infty}(Q)$ such that:

$$
\tilde{k}=\left\{\begin{array}{l}
\frac{\tilde{V}}{\tilde{\Theta}} \text { if } \theta_{1}(\tilde{p})<\theta_{2}(\tilde{p}), \\
0 \text { else }
\end{array} \quad \text { and } l=\left\{\begin{array}{l}
\frac{U}{\Theta} \text { if } \theta_{1}(p)<\theta_{2}(p), \\
0 \text { else }
\end{array}\right.\right.
$$

with $V=v-\theta_{1}, U=u-\theta_{1}$ and $\Theta=\theta_{2}-\theta_{1}$; that way, $0 \leq k \leq 1$ and $0 \leq l \leq 1$ a.e. on $Q$. Then, we introduce the function $\Lambda_{\epsilon}$ defined on $Q \times Q$ through:

$$
\Lambda_{\epsilon}(p, \tilde{p})=\varphi\left(\frac{p+\tilde{p}}{2}\right) \rho_{\epsilon}(p-\tilde{p})
$$

where $\varphi$ is an element of $\mathcal{D}_{+}(] 0, T\left[\times \mathbb{R}^{p}\right)$ and $\rho_{\epsilon}(p-\tilde{p})=\rho_{p, \epsilon}(x-\tilde{x}) \rho_{1, \epsilon}(t-\tilde{t})$.

In entropy relation (9) satisfied by $u$ one chooses $k=\tilde{k}$, the test-function being equal to $\Lambda(., \tilde{p}) \tilde{\Theta}$ and we integrate with respect to $\mathrm{d} \tilde{p}$ over $Q$. The same reasoning leads us to choose the test-function $\Lambda(p,.) \Theta$ and $k=l$, in inequality (9) fulfilled by $v$ and written in the variables $\tilde{p}$ and $\tilde{\sigma}$. Then, we integrate over $Q$ with respect to $\mathrm{d} p$. The two expressions are added up and by taking into account the definition of $\Lambda_{\epsilon}$ it follows:

$$
-I_{1, \epsilon}-I_{2, \epsilon} \leq I_{3, \epsilon}-I_{4, \epsilon} \text { with: }
$$

$$
\begin{aligned}
I_{1, \epsilon} & =\int_{Q \times Q}\left(|U \tilde{\Theta}-\tilde{V} \Theta| \partial_{t} \varphi-\operatorname{sgn}(U \tilde{\Theta}-\tilde{V} \Theta)\{g(p, u) \tilde{\Theta}-g(\tilde{p}, \tilde{v}) \Theta\} \varphi\right) \rho_{\epsilon} \mathrm{d} p \mathrm{~d} \tilde{p} \\
& +\int_{Q \times Q} \operatorname{sgn}(U \tilde{\Theta}-\tilde{V} \Theta)\left\{\{\tilde{\Theta} \mathbf{f}(p, u)-\Theta \mathbf{f}(\tilde{p}, \tilde{v})\}-\left\{\tilde{\Theta} \mathbf{f}\left(p, \tilde{k} \Theta+\theta_{1}\right)-\Theta \mathbf{f}\left(\tilde{p}, l \tilde{\Theta}+\tilde{\theta_{1}}\right)\right\}\right\} \cdot \nabla \varphi \rho_{\epsilon} \mathrm{d} p \mathrm{~d} \tilde{p} \\
& +\int_{Q \times Q} \operatorname{sgn}(U \tilde{\Theta}-\tilde{V} \Theta)\left\{\tilde{\Theta}\left\{\boldsymbol{\nabla} \cdot \mathbf{f}\left(p, \tilde{k} \Theta+\theta_{1}\right)+\partial_{t}\left(\tilde{k} \Theta+\theta_{1}\right)\right\}-\Theta\left\{\tilde{\boldsymbol{\nabla}} \cdot \mathbf{f}\left(\tilde{p}, l \tilde{\Theta}+\tilde{\theta_{1}}\right)+\partial_{\tilde{t}}\left(l \tilde{\Theta}+\tilde{\theta_{1}}\right)\right\}\right\} \Lambda_{\epsilon} \mathrm{d} p \mathrm{~d} \tilde{p}, \\
I_{2, \epsilon}= & \int_{Q \times Q} \operatorname{sgn}(U \tilde{\Theta}-\tilde{V} \Theta)\left\{\tilde{\Theta}\left\{\mathbf{f}(p, u)-\mathbf{f}\left(p, \tilde{k} \Theta+\theta_{1}\right)\right\}+\Theta\left\{\mathbf{f}(\tilde{p}, \tilde{v})-\mathbf{f}\left(\tilde{p}, l \tilde{\Theta}+\tilde{\theta_{1}}\right)\right\}\right\} \cdot \boldsymbol{\nabla} \rho_{\epsilon} \varphi \mathrm{d} p \mathrm{~d} \tilde{p}, \\
I_{3, \epsilon}= & e s s \lim _{\tau \rightarrow 0^{-}}\left(\int_{Q} \int_{\Sigma} \tilde{\Theta} \mathbf{F}\left(u\left(\sigma+\tau \boldsymbol{\nu}, u_{\Gamma}\right) \cdot \boldsymbol{\nu} \Lambda_{\epsilon}(\sigma, \tilde{p}) \mathrm{d} \sigma \mathrm{d} \tilde{p}+\int_{Q} \int_{\Sigma} \Theta \tilde{\mathbf{F}}\left(v(\tilde{\sigma}+\tau \tilde{\boldsymbol{\nu}}), \tilde{v}_{\Gamma}\right) \cdot \tilde{\boldsymbol{\nu}} \Lambda_{\epsilon}(p, \tilde{\sigma}) \mathrm{d} \tilde{\sigma} \mathrm{d} p\right)\right. \\
I_{4, \epsilon}= & \int_{Q} \int_{\Sigma} \tilde{\Theta} \mathbf{F}\left(\tilde{k} \Theta+\theta_{1}, u_{\Gamma}\right) \cdot \boldsymbol{\nu} \Lambda_{\epsilon}(\sigma, \tilde{p}) \mathrm{d} \sigma \mathrm{d} \tilde{p}+\int_{Q} \int_{\Sigma} \Theta \tilde{\mathbf{F}}\left(l \tilde{\Theta}+\tilde{\theta_{1}}, \tilde{v}_{\Gamma}\right) \cdot \tilde{\boldsymbol{\nu}} \Lambda_{\epsilon}(p, \tilde{\sigma}) \mathrm{d} \tilde{\sigma} \mathrm{d} p .
\end{aligned}
$$

We seek to calculate the limit of each integral $I_{i, \epsilon}$ when $\epsilon$ goes to $0^{+}$. For the first two ones the argumentation focuses on the notion of Lebesgue points for an integrable function and uses the Lipschitzian properties of the non-linearities in the same spirit as Kruskov in [12]. For example, let us describe the study of the second term in the first line of $I_{1, \epsilon}$ with typical arguments also used for the second and third lines. Clearly, owing to the 
regularities of functions $g$ and $\theta_{i}$ and to the Lipschitz property for $g$, the second term of the first line in $I_{1, \epsilon}$ has the same limit, when $\epsilon$ goes to $0^{+}$, as the integral:

$$
J_{\epsilon}=\int_{Q \times Q} \operatorname{sgn}(U \tilde{\Theta}-\tilde{V} \Theta) \tilde{\Theta}\{g(p, u)-g(p, v)\} \Lambda_{\epsilon} \mathrm{d} p \mathrm{~d} \tilde{p} .
$$

Accordingly, by arguing whether or not $U \tilde{\Theta}$ is an element of the interval $\mathcal{I}(\Theta \tilde{V} ; \tilde{\Theta} V)$, one proves the existence of a constant $C$, independent from $\epsilon$ such that:

$$
\left|J_{\epsilon}-K_{\epsilon}\right| \leq C \int_{Q \times Q}\{|v-\tilde{v}|+|\Theta-\tilde{\Theta}|\} \Lambda_{\epsilon} \mathrm{d} p \mathrm{~d} \tilde{p}
$$

where,

$$
K_{\epsilon}=\int_{Q \times Q} \operatorname{sgn}(u-v) \tilde{\Theta}\{g(p, u)-g(p, v)\} \Lambda_{\epsilon} \mathrm{d} p \mathrm{~d} \tilde{p} .
$$

So $J_{\epsilon}$ has the same limits as $K_{\epsilon}$, the latter being obtained thanks to the continuity of functions $\theta_{i}$ and $\varphi$.

This reasoning is still valid for the second line of $I_{1, \epsilon}$; but for the third line we also take advantage of the fact that since $\Theta$ is a non-negative function of $W^{1,+\infty}(Q)$, then for a.e. $p_{0}$ of $Q$ such that $\Theta\left(p_{0}\right)=0$, one has $\partial_{t} \Theta\left(p_{0}\right)=0$ and $\nabla \Theta\left(p_{0}\right)=0$. That way, a.e. on $Q$

$$
\operatorname{sgn}(u-v) \partial_{t} \tilde{\Theta}=\operatorname{sgn}(U \tilde{\Theta}-\tilde{V} \tilde{\Theta}) \partial_{t} \tilde{\Theta},
$$

this equality staying true when $\partial_{t} \tilde{\Theta}$ is turned into $\nabla \tilde{\Theta}$. Consequently, it comes:

$$
\begin{aligned}
\lim _{\epsilon \rightarrow 0^{+}} I_{1, \epsilon}=\int_{Q}\left[|u-v| \partial_{t}(\Theta \varphi)+\Theta \mathbf{F}(u, v) \cdot \nabla \varphi-\right. & (\Theta\{g(p, u) \perp g(p, v)\} \\
& \left.\left.+\Theta \sum_{i=1}^{p}\left\{\partial_{x_{i}} f_{i}(p, u) \perp \partial_{x_{i}} f_{i}(p, v)\right\}+\{\omega(u) \perp \omega(v)\}\right) \varphi\right] \mathrm{d} p,
\end{aligned}
$$

with $h(a) \perp h(b)=\operatorname{sgn}(a-b)(h(a)-h(b))$ and $\omega(u)=\partial_{u} \mathbf{f}(p, u)\left(U \nabla \Theta+\Theta \nabla \theta_{1}\right)$.

To study $I_{2, \epsilon}$ we use the regularity of $\mathbf{f}$ to compensate for the term $\boldsymbol{\nabla} \rho_{\epsilon}$ since we must keep in mind that for any integrable function $h$ on $Q$, in all the Lebesgue points $p$ of $h$ :

$$
\lim _{\epsilon \rightarrow 0^{+}} \int_{Q \times Q}|h(p)-h(\tilde{p})|\left|p-\tilde{p} \| \nabla \rho_{\epsilon}(p-\tilde{p})\right| \mathrm{d} p \mathrm{~d} \tilde{p}=0,
$$

this property being valid when the spatial derivatives are turned into the time one. This leads us to decompose $I_{2, \epsilon}$ into the next two integrals:

$$
\begin{aligned}
& I_{21, \epsilon}=\int_{Q \times Q} \operatorname{sgn}(U \tilde{\Theta}-\tilde{V} \Theta)[\tilde{\Theta}-\Theta]\left[\mathbf{f}\left(\tilde{p}, l \tilde{\Theta}+\tilde{\theta_{1}}\right)-\mathbf{f}\left(p, \tilde{k} \Theta+\theta_{1}\right)\right] \cdot \nabla \rho_{\epsilon} \varphi \mathrm{d} p \mathrm{~d} \tilde{p}, \\
& I_{22, \epsilon}=\int_{Q \times Q} \operatorname{sgn}(U \tilde{\Theta}-\tilde{V} \Theta)\left\{\tilde{\Theta}\left[\mathbf{f}(p, u)-\mathbf{f}\left(\tilde{p}, l \tilde{\Theta}+\tilde{\theta_{1}}\right)\right]+\Theta\left[\mathbf{f}(\tilde{p}, \tilde{v})-\mathbf{f}\left(p, \tilde{k} \Theta+\theta_{1}\right)\right]\right\} \cdot \nabla \rho_{\epsilon} \varphi \mathrm{d} p \mathrm{~d} \tilde{p} .
\end{aligned}
$$


Given that $\Theta$ is Lipschitzian on $Q,(13)$ ensures that $I_{21, \epsilon}$ has the same limit as

$$
\int_{Q} \operatorname{sgn}(u-v)[\tilde{\Theta}-\Theta][\mathbf{f}(p, u)-\mathbf{f}(p, v)] \cdot \nabla \rho_{\epsilon} \varphi \mathrm{d} p \mathrm{~d} \tilde{p}
$$

which can be integrated by parts by considering the fact that $\nabla \rho_{\epsilon}(p-\tilde{p})=-\nabla_{\tilde{x}}\left[\rho_{\epsilon}(p-\tilde{p})\right]$. Then, passing to the limit with $\epsilon$ provides:

$$
\lim _{\epsilon \rightarrow 0^{+}} I_{21, \epsilon}=\int_{Q} \nabla \Theta \cdot \mathbf{F}(u, v) \varphi \mathrm{d} p
$$

For $I_{22, \epsilon}$, let us grossly describe the development of the first term, the reasoning being the same for the second one. Thanks to the regularity of $f_{i}$ one has a.e. on $Q \times Q$ and for any $i$ in $\{1, . ., p\}$,

$$
f_{i}(p, u)-f_{i}\left(\tilde{p}, l \tilde{\Theta}+\tilde{\theta_{1}}\right)=\partial_{u} f_{i}(p, u)\left(u-l \tilde{\Theta}-\tilde{\theta_{1}}\right)+\frac{\partial_{u u}^{2} f_{i}(p, \zeta)}{2}\left(u-l \tilde{\Theta}-\tilde{\theta_{1}}\right)^{2}+\int_{\tilde{p}}^{p} \partial_{1} f_{i}\left(\tau, l \tilde{\Theta}-\tilde{\theta_{1}}\right) \mathrm{d} \tau
$$

where $\zeta$ belongs to $\mathcal{I}\left(u, l \tilde{\Theta}+\tilde{\theta}_{1}\right)$.

By noting that $u=l \Theta+\theta_{1}$ a.e. on $Q$ we may refer to (13) and to the Lipschitz property of functions $\theta_{i}$ to pass to the limit in the integrals relative to the three previous terms. Therefore, the first line of $I_{22, \epsilon}$ has the same limit as:

$$
\int_{Q \times Q} \operatorname{sgn}(u-v) \tilde{\Theta}\left[\partial_{u} f_{i}(p, u)\left(l(\Theta-\tilde{\Theta})+\theta_{1}-\tilde{\theta_{1}}\right)+\int_{\tilde{p}}^{p} \partial_{1} f_{i}(\tau, u) d \tau\right] \partial_{x_{i}} \rho_{\epsilon} \varphi \mathrm{d} p \mathrm{~d} \tilde{p} .
$$

An identical procedure for the second term in $I_{22, \epsilon}$ proves that the latter has the same limit as:

$$
\int_{Q \times Q} \operatorname{sgn}(u-v) \tilde{\Theta}\left[\partial_{u} f_{i}(p, v)\left(k(\tilde{\Theta}-\Theta)+\tilde{\theta_{1}}-\theta_{1}\right)+\int_{p}^{\tilde{p}} \partial_{1} f_{i}(\tau, v) d \tau\right] \partial_{x_{i}} \rho_{\epsilon} \varphi \mathrm{d} p \mathrm{~d} \tilde{p} .
$$

Consequently, by adding the two previous integrals, by integrating by parts with respect to $\tilde{x}_{i}$ as for the study of $I_{21, \epsilon}$ and by taking the $\epsilon$-limit, it comes:

$$
\lim _{\epsilon \rightarrow 0^{+}} I_{22, \epsilon}=\int_{Q} \Theta\left[\sum_{i=1}^{p}\left\{\partial_{x_{i}} f_{i}(p, u) \perp \partial_{x_{i}} f_{i}(p, v)\right\}+\{\omega(u) \perp \omega(v)\}\right] \varphi \mathrm{d} x \mathrm{~d} t .
$$

So, to complete the proof of the Kruskov relation (11) we focus on the boundary terms $I_{3, \epsilon}$ and $I_{4, \epsilon}$. In fact, on account of the smoothness of $\Theta$ and $\mathbf{f}, I_{3, \epsilon}$ has the same limit as:

$$
\text { ess } \lim _{\tau \rightarrow 0^{-}}\left(\int_{Q} \int_{\Sigma} \Theta \mathbf{F}\left(u\left(\sigma+\tau \boldsymbol{\nu}, u_{\Gamma}\right) \cdot \boldsymbol{\nu} \Lambda_{\epsilon}(\sigma, \tilde{p}) \mathrm{d} \sigma \mathrm{d} \tilde{p}+\int_{Q} \int_{\Sigma} \Theta \mathbf{F}\left(v(\tilde{\sigma}+\tau \tilde{\boldsymbol{\nu}}), \tilde{v}_{\Gamma}\right) \cdot \tilde{\boldsymbol{\nu}} \Lambda_{\epsilon}(p, \tilde{\sigma}) \mathrm{d} \tilde{\sigma} \mathrm{d} p\right)\right.
$$

and since $\Theta$ is a non-negative function of $W^{1,+\infty}(Q)$, relation (10) ensures that

$$
\text { ess } \lim _{\tau \rightarrow 0^{-}} \int_{\Sigma} \mathbf{F}(u(\sigma+\tau \boldsymbol{\nu}), \varphi) \Theta \delta \mathrm{d} \sigma \text { exists, }
$$


for any $\delta$ of $L_{+}^{1}(\Sigma)$. That is why, by referring to Otto's proof, there exist two functions $\gamma_{u, u_{\Gamma}}$ and $\gamma_{v, v_{\Gamma}}$ in $L^{\infty}(\Sigma)$ such that:

$$
\text { ess } \lim _{\tau \rightarrow 0^{-}} \int_{\Sigma} \mathbf{F}\left(u(\sigma+\tau \boldsymbol{\nu}), u_{\Gamma}\right) \Theta \delta \mathrm{d} \sigma=\int_{\Sigma} \gamma_{u, u_{\Gamma}} \delta \mathrm{d} \sigma \text { and ess } \lim _{\tau \rightarrow 0^{-}} \int_{\Sigma} \mathbf{F}\left(v(\sigma+\tau \boldsymbol{\nu}), v_{\Gamma}\right) \Theta \delta \mathrm{d} \sigma=\int_{\Sigma} \gamma_{v, v_{\Gamma}} \delta \mathrm{d} \sigma .
$$

Moreover,

$$
\lim _{\epsilon \rightarrow 0^{+}} \int_{Q} e s s \lim _{\tau \rightarrow 0^{+}} \int_{\Sigma} \mathbf{F}\left(u(\sigma+\tau \boldsymbol{\nu}), u_{\Gamma}\right) \Theta \Lambda_{\epsilon}(\sigma, \tilde{p}) \mathrm{d} \sigma \mathrm{d} \tilde{p}=\frac{1}{2} \int_{\Sigma} \gamma_{u, u_{\Gamma}} \Theta \varphi \mathrm{d} \sigma
$$

and as this relation holds with $\tilde{v}, \tilde{v}_{\Gamma}$ and $\gamma_{v, v_{\Gamma}}$ it comes:

$$
\lim _{\epsilon \rightarrow 0^{+}} I_{3, \epsilon}=\frac{1}{2} \int_{\Sigma} \Theta\left\{\gamma_{u, u_{\Gamma}}+\gamma_{v, v_{\Gamma}}\right\} \varphi \mathrm{d} \sigma
$$

It is the same for $I_{4, \epsilon}$, since the regularities of $\mathbf{f}$ and $\Theta$ ensure, for example, that the first term in $I_{4, \epsilon}$ has the same limit as the integrals

$$
\int_{Q} \int_{\Sigma} \Theta \mathbf{F}\left(\tilde{v}, u_{\Gamma}\right) \cdot \boldsymbol{\nu} \Lambda_{\epsilon}(\sigma, \tilde{p}) \mathrm{d} \sigma \mathrm{d} \tilde{p}+\int_{Q} \int_{\Sigma} \Theta \mathbf{F}\left(u, \tilde{v}_{\Gamma}\right) \cdot \tilde{\boldsymbol{\nu}} \Lambda_{\epsilon}(\tilde{\sigma}, p) \mathrm{d} \tilde{\sigma} \mathrm{d} p .
$$

That way, there exist two functions $\gamma_{v, u_{\Gamma}}$ and $\gamma_{u, v_{\Gamma}}$ in $L^{\infty}(\Sigma)$ such that:

$$
\lim _{\epsilon \rightarrow 0^{+}} I_{4, \epsilon}=\frac{1}{2} \int_{\Sigma} \Theta\left\{\gamma_{v, u_{\Gamma}}+\gamma_{u, v_{\Gamma}}\right\} \varphi \mathrm{d} \sigma .
$$

Finally, adding up limits (12),(14),(15),(16) and (17) results in:

$$
-\int_{Q}\left(|u-v| \partial_{t}(\Theta \varphi)+\mathbf{F}(u, v) \cdot \nabla(\Theta \varphi)-\{g(p, u) \perp g(p, v)\} \theta \varphi\right) \mathrm{d} p \leq \frac{1}{2} \int_{\Sigma}\left(\gamma_{u, u_{\Gamma}}-\gamma_{u, v_{\Gamma}}+\gamma_{v, v_{\Gamma}}-\gamma_{v, u_{\Gamma}}\right) \Theta \varphi \mathrm{d} \sigma
$$

for any function $\varphi$ of $\mathcal{D}_{+}(] 0, T\left[\times \mathbb{R}^{p}\right)$.

By density this inequality is still fulfilled for all functions $\varphi$ such that $\Theta \varphi$ is a positive element of $W^{1,1}(Q)$ with $\Theta(0,.) \varphi(0,)=.\Theta(T,.) \varphi(T,)=$.0 . So, one may consider the following test-function:

$$
\varphi(t, x)=\varphi_{\mu}(t, x)=\left\{\begin{array}{l}
\frac{\xi(t)}{\Theta(t, x)} \text { if } \Theta(t, x)>\mu, \\
\frac{\xi(t)}{\mu} \text { else }
\end{array}\right.
$$

$\xi$ being any element of $W_{+}^{1,1}(0, T)$ and $\mu$ a strictly positive real $\left(\mu<\|\Theta\|_{\infty}\right)$. We thus split the integration field into $[0<\Theta \leq \mu]$ and $[\mu<\Theta]$. So as to calculate the limit when $\mu$ goes to $0^{+}$for the left-hand member of (18) on $[0<\Theta \leq \mu]$ we note that:

$$
\frac{1}{\mu}|u-v| \leq 1 \text { a.e. on }[\Theta \leq \mu] \text {, and } \lim _{\mu \rightarrow 0^{+}} \int_{[0<\Theta \leq \mu]} \partial_{t} \Theta \mathrm{d} p=0, \lim _{\mu \rightarrow 0^{+}} \int_{[0<\Theta \leq \mu]} \nabla \Theta \mathrm{d} p=0 .
$$


That way,

$$
\lim _{\mu \rightarrow 0^{+}} \frac{1}{\mu} \int_{[0<\Theta \leq \mu]}\left(|u-v| \partial_{t}(\Theta \zeta)+\mathbf{F}(u, v) \cdot \nabla \Theta \zeta-\{g(p, u) \perp g(p, v)\} \Theta \zeta\right) \mathrm{d} p=0
$$

For the right-hand term of (18), we observe that for any real $a, b, c, d$ and for any $i$ of $\{1, \ldots, p\}$,

$$
\left|F_{i}(a, c)-F_{i}(a, d)+F_{i}(b, c)-F_{i}(b, d)\right| \leq 2 A_{i}|c-d|
$$

where $A_{i}$ is the Lipschitz constant of $f_{i}$ with respect to its third variable, uniformly in $(t, x)$. Thus, when $a=u(\sigma+\tau \boldsymbol{\nu}), b=v(\sigma+\tau \boldsymbol{\nu}), c=u_{\Gamma}$ and $d=v_{\Gamma}$, the limit, when $\tau$ goes to $0^{-}$outside of a set of measure zero, provides:

$$
\int_{\Sigma}\left(\gamma_{u, u_{\Gamma}}-\gamma_{u, v_{\Gamma}}+\gamma_{v, v_{\Gamma}}-\gamma_{v, u_{\Gamma}}\right) \Theta \varphi \mathrm{d} \sigma \leq 2 A \int_{\Sigma}\left|u_{\Gamma}-v_{\Gamma}\right| \Theta \varphi \mathrm{d} \sigma
$$

This inequality and the fact that $\left|u_{\Gamma}-v_{\Gamma}\right| \leq \mu$ a.e on $\Sigma$ prove that the contribution on $[0<\Theta \leq \mu]$ of the right-hand side term of (18) goes to zero with $\mu$.

Eventually, since the Lebesgue-dominated convergence theorem gives the limit on the subdomain $[\mu<\Theta]$ when $\mu$ goes to $0^{+}$, one has:

$$
\left.-\int_{Q}|u-v| \partial_{t} \zeta \mathrm{d} p \leq A \int_{\Sigma}\left|u_{\Gamma}-v_{\Gamma}\right| \zeta \mathrm{d} \sigma+\int_{Q}\{g(p, u) \perp g(p, v)\}\right) \zeta \mathrm{d} p
$$

for any function $\zeta$ of $W_{+}^{1,1}(0, T)$. Now the conclusion is classical: it uses the Lipschitz condition for $g$ and a piecewise linear approximation of $\mathbb{I}_{0, t}, t$ being given outside a of a set of measure zero. Thanks to the definition of initial condition (7) for $u$ and $v$ and to the Gronwall lemma we complete the proof of Theorem 2.1.

\section{EXISTENCE VIA AN ENTROPY PROCESS SOLUTION}

The method of penalization is applied with a view to obtaining an existence result. Hence, for each value of the strictly positive parameter $\eta$ - intended to go to zero - the following regularized problem is introduced: find a measurable function $u_{\eta}$ such that

$$
\begin{gathered}
\mathbb{H}\left(t, x, u_{\eta}\right)+\frac{1}{\eta} \beta_{\eta}\left(t, x, u_{\eta}\right)=0 \text { on } Q, \\
u_{\eta}=u_{\Gamma, \eta} \text { on (a part of) } \Sigma, u_{\eta}(0, .)=u_{0, \eta} \text { on } \Omega
\end{gathered}
$$

where $\beta_{\eta}(t, x, u)=-\left(u-\theta_{1, \eta}(t, x)\right)^{-}+\left(u-\theta_{2, \eta}(t, x)\right)^{+}$and $u_{\Gamma, \eta}$ and $u_{0, \eta}$ are the standard regularizations of $u_{\Gamma}$ and $u_{0}$ by means of mollifer sequences indexed on $\eta$. Moreover, $\theta_{i, \eta}, i=1,2$ is obtained through changing $\theta_{i}$ in its spatial regularization.

\subsection{Study of the penalized problem}

We need estimates of $u_{\eta}$ that are independent from $\eta$. But as the penalizing operator $\beta_{\eta}$ depends on time and space variables via obstacle functions $\theta_{i}$ one may even estimate the uniform bound of $u_{\eta}$. Indeed, the next statement holds: 
Property 3.1. For any strictly positive real $\eta$ :

$$
\begin{gathered}
\left\|u_{\eta}(t, .)\right\|_{L^{\infty}(\Omega)} \leq M(t) \text { for any } t \text { of }[0, T], \\
\frac{1}{\eta}\left\|\beta_{\eta}\left(t, x, u_{\eta}\right)\right\|_{L^{1}(Q)} \leq C
\end{gathered}
$$

where $M$ is given by (4) and $C$ is a constant independent from any parameter.

Proof. According to the works of Bardos et al. [2], $\eta$ being fixed, the penalized problem (19) - (20) has a unique weak entropy solution $u_{\eta}$ in $B V(Q) \cap L^{\infty}(Q)$. In addition, when $\epsilon$ goes to $0^{+}, u_{\eta}$ is the $L^{q}(Q)$ and $\mathcal{C}\left([0, T] ; L^{1}(\Omega)\right)$-limit, $1 \leq q<+\infty$, of the sequence $\left(u_{\epsilon, \eta}\right)_{\epsilon>0}$ defined for any $\epsilon$ by: find $u_{\epsilon, \eta}$ in $H^{2}(Q) \cap L^{\infty}(Q)$, such that:

$$
\begin{aligned}
& \mathbb{H}\left(t, x, u_{\epsilon, \eta}\right)+\frac{1}{\eta} \beta_{\eta}\left(t, x, u_{\epsilon, \eta}\right)=\epsilon \Delta u_{\epsilon, \eta} \text { a.e. on } Q, \\
& u_{\epsilon, \eta}=u_{\Gamma, \eta} \text { a.e. on } \Sigma, u_{\epsilon, \eta}(0, .)=u_{0, \eta} \text { a.e. on } \Omega .
\end{aligned}
$$

In fact, $(21)$ is a consequence of the maximum principle for $u_{\epsilon, \eta}$ and of the monotony of the penalizing operator $w \rightarrow \beta_{\eta}(t, x, w)$. Nevertheless, we develop the demonstration with typical arguments used in the sequel. So, $\lambda$ being any strictly positive real, we note $\operatorname{sgn}_{\lambda}$ the approximation of the function "sgn" defined, for any positive real $x$, through:

$$
\operatorname{sgn}_{\lambda}(x)=\min \left(\frac{x}{\lambda}, 1\right) \text { and } \operatorname{sgn}_{\lambda}(-x)=-\operatorname{sgn}_{\lambda}(x)
$$

Moreover, we denote

$$
I_{\lambda}(x)=\int_{0}^{x} \operatorname{sgn}_{\lambda}(\tau) \mathrm{d} \tau .
$$

Then, $t$ being given in $] 0, T]$, we consider the $L^{2}(] 0, t[\times \Omega)$-scalar product between the diffusion equation (23) and the test-function $\operatorname{sgn}_{\lambda}\left(u_{\epsilon, \eta}-M(t)\right)^{+}$. By taking into account the definition of $M(t)$ and the fact that $\beta_{\eta}\left(t, x, u_{\epsilon, \eta}\right) \operatorname{sgn}_{\lambda}\left(u_{\epsilon, \eta}-M(t)\right)^{+} \geq \beta_{\eta}(t, x, M(t)) \operatorname{sgn}_{\lambda}\left(u_{\epsilon, \eta}-M(t)\right)^{+} \geq 0$ a.e. on $Q$, some integrations by parts in time and space give the next inequality:

$$
\begin{aligned}
& \int_{\Omega} I_{\lambda}\left(u_{\epsilon, \eta}(t, x)-M(t)\right)^{+} \mathrm{d} x+\int_{0}^{t} \int_{\Omega} \mathbb{H}(t, x, M) \operatorname{sgn}_{\lambda}\left(u_{\epsilon, \eta}-M\right)^{+} \mathrm{d} x \mathrm{~d} t \\
& \leq \int_{0}^{t} \int_{\Omega}\left\{\mathbf{f}\left(t, x, u_{\epsilon, \eta}\right)-\mathbf{f}(t, x, M)\right\} \cdot \nabla \operatorname{sgn}_{\lambda}\left(u_{\epsilon, \eta}-M\right)^{+} \mathrm{d} x \mathrm{~d} t+M_{g}^{\prime} \int_{0}^{t} \int_{\Omega}\left(u_{\epsilon, \eta}-M\right)^{+} \mathrm{d} x \mathrm{~d} t .
\end{aligned}
$$

The Sacks lemma shows that the first term of the right-hand member goes to zero when $\lambda$ tends to $0^{+}$. Thus, as a result of the Gronwall lemma, one may conclude the proof of Property 3.1 ensuring that $\mathbb{H}(t, x, M)$ is non-negative a.e. on $Q$. For this purpose, we remark that:

$$
M^{\prime}(t)=C_{1} M(t)+\max _{[0, T] \times \bar{\Omega}}|\mathbb{H}(t, x, 0)|
$$

and owing to the Lipschitz properties for $g$ and $\mathbf{f}$,

$$
\mathbb{H}(t, x, M(t)) \geq-C_{1} M(t)+\mathbb{H}(t, x, 0)+M^{\prime}(t) .
$$


Accordingly, the $\lambda$-limit provides the majoration for $u_{\epsilon, \eta}$

$$
u_{\epsilon, \eta}(t, x) \leq M(t) \text { a.e. in } Q,
$$

and the minoration is obtained through the same $L^{1}$-cut off method. Estimate (21) follows by passing to the limit with respect to $\epsilon$.

We now seek an estimate of the penalized term in (23). With this view we first consider firstly the $L^{2}(Q)$ scalar product between $(23)$ and the test-function $-\operatorname{sgn}_{\lambda}\left(u_{\epsilon, \eta}-\theta_{1, \eta}\right)^{-}$. The next inequality comes:

$$
\begin{array}{r}
\frac{1}{\eta} \int_{Q}\left(u_{\epsilon, \eta}-\theta_{1, \eta}\right)^{-} \operatorname{sgn}_{\lambda}\left(u_{\epsilon, \eta}-\theta_{1, \eta}\right)^{-} \mathrm{d} x \mathrm{~d} t+\int_{Q}\left\{\mathbf{f}\left(t, x, u_{\epsilon, \eta}\right)-\mathbf{f}\left(t, x, \theta_{1, \eta}\right)\right\} \cdot \nabla \operatorname{sgn}_{\lambda}\left(u_{\epsilon, \eta}-\theta_{1, \eta}\right)^{-} \mathrm{d} x \mathrm{~d} t \\
\leq \int_{Q}\left\{\left|\mathbb{H}\left(t, x, \theta_{1, \eta}\right)\right|+\epsilon\left|\Delta \theta_{1, \eta}\right|+M_{g}^{\prime}\left(u_{\epsilon, \eta}-\theta_{1, \eta}\right)^{-}\right\} \mathrm{d} x \mathrm{~d} t
\end{array}
$$

As previously, the Sacks lemma shows that the second term in the right-hand member goes to zero with $\lambda$ and the left-hand one is bounded by a constant $C$ independent from any parameter (due to the regularities of $\theta_{1, \eta}$ some majorations of the time and space derivatives of $\theta_{1, \eta}$, independent from $\eta$, hold). That way, the $\lambda$-limit and then, the $\epsilon$-limit provide the existence of a constant $C$ such that:

$$
\frac{1}{\eta} \int_{Q}\left(u_{\eta}-\theta_{1, \eta}\right)^{-} \mathrm{d} x \mathrm{~d} t \leq C
$$

Secondly, the $L^{2}(Q)$-scalar product between $(23)$ and the test-function $\operatorname{sgn}_{\lambda}\left(u_{\epsilon, \eta}-\theta_{2, \eta}\right)^{+}$allows us to conclude the proof of Property 3.1 .

To study the behaviour of the sequence $\left(u_{\eta}\right)_{\eta>0}$ that is to pass to the $\eta$-limit in the nonlinear terms of $\mathbb{H}$ we must refer to properties of bounded sequences in $L^{\infty}$.

\subsection{Entropy process solution}

Let $\mathcal{O}$ be an open bounded subset of $\mathbb{R}^{q}(q \geq 1)$ and let $\left(u_{n}\right)_{n>0}$ be a bounded sequence in $L^{\infty}(\mathcal{O})$. Clearly, for any continuous function $h$, there exists $\bar{h}$ in $L^{\infty}(Q)$ such that, for a subsequence,

$$
h\left(u_{n}\right) \rightarrow \bar{h} \text { weakly in } L^{\infty}(\mathcal{O}) .
$$

Since the works of Tartar [17] and Ball [1], one has been able to describe the composite limit $\bar{h}$. Actually, thanks to the properties for weak-* topology on the space of Radon measures the next compacity result holds:

Property 3.2. Let $\left(u_{n}\right)_{n>0}$ be a sequence of measurable functions on $\mathcal{O}$ such that:

$$
\exists M>0, \forall n>0,\left\|u_{n}\right\|_{L^{\infty}(\mathcal{O})} \leq M .
$$

Then, there exists a subsequence $\left(u_{\varphi(n)}\right)_{n>0}$ and $\left(\nu_{w}\right)_{w \in \mathcal{O}}$ a family of probability measures on $\mathbb{R}$, with support in $[-M, M]$, such that, for all continuous bounded functions $h$ on $\mathcal{O} \times]-M, M\left[\right.$, the sequence $\left(h\left(., u_{\varphi(n)}\right)\right)_{n>0}$ converges in $L^{\infty}(\mathcal{O})$ weak-* towards the element:

$$
w \rightarrow \int_{\mathbb{R}} h(x, \lambda) \mathrm{d} \nu_{w}(\lambda) .
$$

The mapping $\nu: w \rightarrow \nu_{w}$ is called "a Young measure associated with the sequence $\left(u_{n}\right)_{n>0}$ ". 
Such a result has found its first application in [17] and [8] for the approximation through the artificial viscosity method of the Cauchy problem in $\mathbb{R}^{p}$ for a scalar conservation law, as one can establish a uniform $L^{\infty}$-control of approximate solutions. It has also been applied to the numerical analysis of transport equations $[6,7,9,11,19]$ since "Finite-Volume" schemes only give an $L^{\infty}$-estimate uniformly with respect to the mesh length of the numerical solution. Lastly, it has been extended to the Dirichlet problem for scalar conservation laws by introducing the notion of Young measure-trace $[3,15,16,18]$.

As a matter of fact, the properties for the generalized inverse of the distribution function linked to a probability measure permits to turn the integration with respect to the measure $d \nu_{w}$ into an integration with respect to the Lebesgue measure on ]0,1[, which can be permuted with the integration on $\mathcal{O}$. Indeed, owing to Eymard et al. [9], the next statement holds:

Property 3.3. Let $w \rightarrow \nu_{w}$ be a Young measure with support in $[-M, M]$. There exists a function $\pi$ in $L^{\infty}(] 0,1[\times \mathcal{O})$ such that for all continuous bounded functions $h$ on $\left.\mathcal{O} \times\right]-M, M[$ :

$$
\int_{\mathbb{R} \times \mathcal{O}} h(x, \lambda) \mathrm{d} \nu_{w}(\lambda) \mathrm{d} x=\int_{0,1[\times \mathcal{O}} h(x, \pi(\alpha, w)) \mathrm{d} \alpha \mathrm{d} x \text { for a.e. } w \text { in } \mathcal{O} .
$$

Here, we adapt this concept when the approximating sequence $\left(u_{\eta}\right)_{\eta>0}$ is the sequence of weak entropy solutions to penalized problems. As a result, a priori estimates of Property 3.1 allow us to announce the main result of this section:

Theorem 3.1. There exists an entropy process solution to obstacle problem (1),(2),(3) in the sense that there exists a function $\pi$ of $L^{\infty}(] 0,1[\times Q)$ such that:

i) for a.e. $(\alpha, t, x)$ in $] 0,1[\times Q$

$$
\theta_{1}(t, x) \leq \pi(\alpha, t, x) \leq \theta_{2}(t, x),
$$

ii) for all functions $\xi$ of $\mathcal{D}_{+}(] 0, T[\times \Omega)$, for any real $k$ of $[0,1]$,

$$
\int_{0,1[\times Q} \mathcal{L}(\pi, K, \xi) \mathrm{d} \alpha \mathrm{d} x \mathrm{~d} t \geq 0
$$

with $K=k\left(\theta_{2}-\theta_{1}\right)+\theta_{1}$.

iii)

$$
\text { ess } \lim _{t \rightarrow 0^{+}} \int_{0,1[\times \Omega}\left|\pi(\alpha, t, x)-u_{0}(x)\right| \mathrm{d} \alpha \mathrm{d} x=0,
$$

iv) for any real $k$ of $[0,1]$ and for all functions $\zeta$ of $L_{+}^{1}(\Sigma)$,

$$
\text { ess } \lim _{\tau \rightarrow 0^{-}} \int_{] 0,1[\times \Sigma} \mathcal{F}\left(\pi(\alpha, \sigma+\tau \boldsymbol{\nu}), u_{\Gamma}, K\right) \cdot \boldsymbol{\nu} \zeta \mathrm{d} \sigma \geq 0 .
$$

Proof. Further to (21) there exists a subsequence extracted from $\left(u_{\eta}\right)_{\eta>0}-$ still labeled $\left(u_{\eta}\right)_{\eta>0}$ - and a measurable and bounded function $\pi$ on $] 0,1[\times Q$ such that for any continuous bounded function $\psi$ on $Q \times]-M, M[$,

$$
\lim _{\eta \rightarrow 0^{+}} \int_{Q} \psi\left(t, x, u_{\eta}\right) \varphi \mathrm{d} x \mathrm{~d} t=\int_{] 0,1[\times Q} \psi(t, x, \pi(\alpha, t, x)) \varphi \mathrm{d} \alpha \mathrm{d} x \mathrm{~d} t,
$$


for any function $\varphi$ of $L^{1}(Q)$.

So, (24) follows from (22) and in order to prove (25), (26), (27) we come back to the viscous equation (23). We introduce a boundary entropy-entropy flux pair in the sense of Otto [14], that means a pair of $(H, \mathbf{Q})$ $\mathcal{C}^{2}\left(\mathbb{R}^{2}\right)$-class functions such that for any $w$ of $\mathbb{R},(H(., w), \mathbf{Q}(., w))$ is an entropy-entropy flux pair for $\mathbb{H}$ :

$$
z \rightarrow H(z, w) \text { is convex and } \mathbf{Q}(z, w)=\int_{w}^{z} \partial_{1} H(\tau, w) \partial_{u} \mathbf{f}(t, x, \tau) \mathrm{d} \tau .
$$

In addition, $(H, \mathbf{Q})$ satisfies

$$
H(w, w)=0, \mathbf{Q}(w, w)=0, \partial_{1} H(w, w)=0,
$$

where $\partial_{1} H$ denotes the partial derivative of $H$ with respect to its first variable.

To simplify the writing we drop the indexes $\epsilon$ and $\eta$ temporarily. Thus, due to the convexity of $H(., w)$ for any $w$ of $\mathbb{R}$ and since $\theta_{1} \leq K \leq \theta_{2}$ on $Q$,

$$
\partial_{1} H(u, K) \Delta u \leq \boldsymbol{\nabla} \cdot\left[\partial_{1} H(u, K) \nabla u\right]-\partial_{21}^{2} H(u, K) \nabla u \cdot \nabla K \text { and } 0 \leq \partial_{1} H(u, K) \beta(t, x, u) \text { a.e. on } Q .
$$

and if we transform the transport term into

$$
\partial_{1} H(u, K) \boldsymbol{\nabla} \cdot \mathbf{f}(t, x, u)=\boldsymbol{\nabla} \cdot \mathbf{Q}(u, K)+\int_{K}^{u}\left\{\partial_{u} \mathbf{f}(t, x, \tau) \partial_{12}^{2} H(\tau, K) \cdot \nabla K-\boldsymbol{\nabla} \cdot \mathbf{f}(t, x, \tau) \partial_{11}^{2} H(\tau, K)\right\} \mathrm{d} \tau .
$$

The multiplication of (23) by $\partial_{1} H(u, K)$ ensures that a.e. on $Q$,

$$
\partial_{t} H(u, K)+\boldsymbol{\nabla} \cdot \mathbf{Q}(u, K)+G(u, K) \leq \epsilon\left(\boldsymbol{\nabla} \cdot\left[\partial_{1} H(u, K) \boldsymbol{\nabla} u\right]-\partial_{21}^{2} H(u, K) \nabla u \cdot \nabla K\right),
$$

where

$$
\begin{aligned}
G(u, K)=\int_{K}^{u}\left\{\partial_{u} \mathbf{f}(t, x, \tau) \partial_{12}^{2} H(\tau, K) \cdot \nabla K-\nabla \cdot \mathbf{f}(t, x, \tau) \partial_{11}^{2} H(\tau, K)\right\} & \mathrm{d} \tau \\
& \quad-\partial_{2} H(u, K) \partial_{t} K+g(t, x, u) \partial_{1} H(u, K) .
\end{aligned}
$$

Now let us consider the $L^{2}(Q)$-scalar product between $(28)$ and a test-function $\xi$ of $\mathcal{D}_{+}(]-\infty, T[\times \Omega)$. After integrating by parts one may have $\epsilon$ tend to $0^{+}$by referring to the convergence properties of the sequence $\left(u_{\epsilon, \eta}\right)_{\epsilon>0}$. It provides a regularized weak entropy formulation for the solution $u_{\eta}$ of penalized problem (19)-(20), in which we can pass to the limit with $\eta$ as all the non-linearities are continuous with respect to $\eta$-depending functions. Hence, it comes:

$$
-\int_{0,1[\times Q}\left(H(\pi, K) \partial_{t} \xi+\mathbf{Q}(u, K) \cdot \nabla \xi-G(\pi, K) \xi\right) \mathrm{d} \alpha \mathrm{d} x \mathrm{~d} t \leq \int_{\Omega} H\left(u_{0}, K(0, x)\right) \xi(0, x) \mathrm{d} x .
$$

In the particular situation when, for any $l$ of $\mathbb{N}$,

$$
H(z, w)=H_{l}(z, w)=\left((z-w)^{2}+\left(\frac{1}{l}\right)^{2}\right)^{1 / 2}-\frac{1}{l}
$$


the limit when $l$ goes to $+\infty$ provides:

$$
-\int_{] 0,1[\times Q} \mathcal{L}(\pi, K, \zeta) \mathrm{d} \alpha \mathrm{d} x \mathrm{~d} t \leq \int_{\Omega}\left|u_{0}-K(0, x)\right| \xi(0, x) \mathrm{d} x .
$$

Thus, one gets (25). Moreover, Otto's reasoning in [14] developed for an homogeneous scalar conservation law can be adapted to the present context where $\mathbb{H}$ presents a reaction term and $K$ is a time and space depending function. Hence, we may be sure that if the previous inequality holds then for any function $\varphi$ of $L^{\infty}(\Omega)$, $0 \leq \varphi \leq 1$ a.e. on $\Omega$,

$$
\begin{aligned}
\text { ess } \lim _{t \rightarrow 0^{+}} \sup \int_{0,1[\times \Omega} \mid \pi(\alpha, t, x)-\varphi(x)\left(\theta_{2}(0, x)-\theta_{1}(0, x)\right)- & \theta_{1}(0, x) \mid \mathrm{d} \alpha \mathrm{d} x \\
& \leq \int_{\Omega}\left|u_{0}(x)-\varphi(x)\left(\theta_{2}(0, x)-\theta_{1}(0, x)\right)-\theta_{1}(0, x)\right| \mathrm{d} x .
\end{aligned}
$$

So initial condition (26) is obtained by choosing:

$$
\varphi(x)=\left\{\begin{array}{l}
\frac{u_{0}(x)}{\theta_{2}(0, x)-\theta_{1}(0, x)}-\theta_{1}(0, x) \text { if } \theta_{1}(0, x)<\theta_{2}(0, x), \\
0 \text { else. }
\end{array}\right.
$$

The demonstration of Dirichlet boundary condition (27) for $\pi$ also refers to the Otto's basic proof which, for any $\epsilon>0$, introduces the function $\zeta$ defined by

$$
\zeta(x)=1-\exp \left(-\frac{A+\epsilon L}{\epsilon} s(x)\right)
$$

where $A$ is the Lipschitz constant of $\mathbf{f}$ with respect to its third variable, uniformly with respect to $(t, x)$ and for any strictly positive parameter $\delta$,

$$
s(x)=\left\{\begin{array}{l}
\min (\operatorname{dist}(x, \Gamma), \delta) \text { for } x \in \Omega \\
-\min (\operatorname{dist}(x, \Gamma), \delta) \text { for } x \in \mathbb{R}^{p} \backslash \Omega,
\end{array}\right.
$$

with $L=\sup _{0<s(x)<\delta}|\Delta s(x)|$. That way, for any $\varphi$ of $W_{+}^{1,1}\left(\mathbb{R}^{p}\right)$,

$$
A \int_{\Omega}|\nabla \zeta| \varphi \mathrm{d} x \leq \epsilon \int_{\Omega} \nabla \zeta \cdot \nabla \varphi \mathrm{d} x+(A+L \epsilon) \int_{\Gamma} \varphi \mathrm{d} \lambda .
$$

Consequently, we take the $L^{2}(Q)$-scalar product between (28) and the test-function $\zeta \xi$, in which $\xi$ is any element of $\mathcal{D}_{+}(] 0, T\left[\times \mathbb{R}^{p}\right)$. Then, some integrations by parts give:

$$
\begin{aligned}
-\int_{Q}\left(H(u, K) \zeta \partial_{t} \xi+\right. & \mathbf{Q}(u, K) \cdot \nabla \xi \zeta-G(u, K) \zeta \xi) \mathrm{d} x \mathrm{~d} t \\
& \leq \int_{Q} \mathbf{Q}(u, K) \cdot \nabla \zeta \xi \mathrm{d} x \mathrm{~d} t-\epsilon \int_{Q}\left\{\partial_{1} H(u, K) \nabla u \cdot \nabla(\xi \zeta)+\partial_{12}^{2} H(u, K) \nabla u \cdot \nabla K \xi \zeta\right\} \mathrm{d} x \mathrm{~d} t .
\end{aligned}
$$


In the right-hand member of the above inequality we consider the fact that

$$
|\mathbf{Q}(u, K)| \leq A H(u, K) \text { a.e. on } Q,
$$

and we use the weak differential inequality for $\zeta$, with $\varphi=H(u, K) \xi$. As $\zeta$ goes to 1 in $L^{1}(\Omega)$ and $\epsilon \boldsymbol{\nabla} \zeta$ goes to 0 in $\left(L^{1}(\Omega)\right)^{p}$ one may successively pass to the limit when $\epsilon$ and $\eta$ tend to $0^{+}$. For any boundary entropy-entropy flux pair, it comes:

$$
-\int_{0,1[\times Q}\left\{H(\pi, K) \partial_{t} \xi+\mathbf{Q}(\pi, K) \cdot \nabla \xi-G(\pi, K) \xi\right\} \mathrm{d} \alpha \mathrm{d} x \mathrm{~d} t \leq A \int_{\Sigma} H\left(u_{\Gamma}, K\right) \xi \mathrm{d} \sigma .
$$

Thus, when one refers to Otto's works and uses the smoothness of functions $\mathbf{f}$ and $\theta_{i}$, this inequality implies that for any $\varphi$ of $L_{+}^{\infty}(\Sigma), 0 \leq \varphi \leq 1$ a.e. on $\Sigma$, and $\delta$ of $L_{+}^{1}(\Gamma)$,

$$
\text { ess } \lim _{\tau \rightarrow 0^{-}} \int_{\Sigma} \mathbf{Q}\left(\pi(\alpha, \sigma+\tau \boldsymbol{\nu}), \varphi\left(\theta_{2}-\theta_{1}\right)+\theta_{1}\right) \delta \mathrm{d} \alpha \mathrm{d} \sigma \geq-A \int_{\Sigma} H\left(u_{\Gamma}, \varphi\left(\theta_{2}-\theta_{1}\right)+\theta_{1}\right) \delta \mathrm{d} \sigma .
$$

To conclude, let us consider

$$
\varphi(\sigma)=\left\{\begin{array}{l}
\frac{u_{\Gamma}}{\theta_{2}-\theta_{1}}-\theta_{1} \text { if } \theta_{1}(\sigma)<\theta_{2}(\sigma) \\
0 \text { else }
\end{array}\right.
$$

and for any $l$ of $\mathbb{N}$ and $k$ of $\mathbb{R}$,

$$
H(z, w)=H_{l}(z, w)=\left((\operatorname{dist}(z, \mathcal{I}[w, k]))^{2}+\left(\frac{1}{l}\right)^{2}\right)^{1 / 2}-\frac{1}{l},
$$

that way, $\left(\mathbf{Q}_{l}\right)_{l \in \mathbb{N}}$ converges uniformly as $l$ goes to $+\infty$, to $\mathcal{F}(z, w, k)$. Boundary condition (27) follows, which completes the proof of Theorem 3.1.

\subsection{Consequences}

Let $\pi$ and $\omega$ be two entropy process solutions of obstacle problem (1)-(3) in the sense of (24)-(27). Then, as Kruskov relation (11) obviously remains valid for in the context of entropy process, one has for a.e. $t$ of $] 0, T[$ :

$$
\int_{0}^{t} \int_{Q \times] 0,1\left[^{2}\right.}|\pi(\alpha, t, x)-\omega(\beta, t, x)| \mathrm{d} \alpha \mathrm{d} \beta \mathrm{d} x \mathrm{~d} t=0 .
$$

Therefore, as mentioned in [9], the two processes $\pi$ and $\omega$ are equal, for a.e. $(t, x)$ on $Q$, to a common value $u(t, x)$, which does not depend on $\alpha$ or $\beta$ in ]0,1[. In addition, $u$ is a measurable and bounded function on $Q$. It can also be understood that for a.e. $(t, x)$ in $Q$, the probability measures $d \mu_{(t, x)}$ and $d \varpi_{(t, x)}$ associated with $\pi$ and $\omega$ thanks to Property 3.2 have a punctual support and thus are equal to a Dirac mass centered on a point noted $u(t, x)(c f$. [8]). Since relations $(24),(25),(26)$ and (27) are now respectively written under the form $(5),(6),(7)$ and $(8)$, we are able to announce:

Theorem 3.2. Obstacle problem (1), (2), (3) has a unique weak entropy solution characterized through the formulation of Definition 1.1.

To conclude, let us keep in mind that this uniqueness property ensures that $u$ is the limit in $L^{r}(Q), 1 \leq r<$ $+\infty$, and a.e. on $Q$, of the whole sequence of approximate solutions (see for example [8]). 


\section{Some Properties for the Bilateral obstacle Problem}

We now take advantage of the strong convergence in $L^{r}, 1 \leq r<+\infty$, of the penalized sequence $\left(u_{\eta}\right)_{\eta>0}$ toward the weak entropy solution $u$ of $(1),(2),(3)$ first to provide first some sensitivity properties of $u$ with respect to the associated obstacle functions $\theta_{i}$. These results are obtained thanks to the monotony of the penalizing operator $w \rightarrow \beta(t, x, w)$, which guarantees that (with the notations introduced in Th. 3.1) :

Lemma 4.1. Let $u$ and $v$ be two weak entropy solutions to obstacle problem $(1),(2),(3)$ associated respectively with boundary conditions $\left(u_{0}, u_{\Gamma}\right)$ and $\left(v_{0}, v_{\Gamma}\right)$ and corresponding to obstacle functions $\left(\theta_{1}, \theta_{2}\right)$ and $\left(\psi_{1}, \psi_{2}\right)$. Let us assume that $\theta_{1} \leq \psi_{1} \leq \theta_{2} \leq \psi_{2}$ a.e. on $Q$. Then, for a.e. $t$ of $] 0, T[$,

$$
\int_{\Omega}(u(t, x)-v(t, x))^{+} \mathrm{d} x \leq\left(A \int_{0}^{t} \int_{\Gamma}\left(u_{\Gamma}-v_{\Gamma}\right)^{+} \mathrm{d} \sigma+\int_{\Omega}\left(u_{0}-v_{0}\right)^{+} \mathrm{d} x\right) \mathrm{e}^{M_{g}^{\prime} t} .
$$

Proof. By coming back to the viscous equation (23) for $u$ and $v$, we obtain a.e. on $Q$ (we have dropped indexes $\epsilon$ and $\eta$ temporarily):

$$
\mathbb{H}(t, x, u)-\mathbb{H}(t, x, v)+\frac{1}{\eta}\left(\beta(t, x, u)-\beta^{*}(t, x, v)\right)=\epsilon \Delta(u-v),
$$

where $\beta^{*}(t, x, v)=-\left(v-\psi_{1}\right)^{-}+\left(v-\psi_{2}\right)^{+}$.

Given $t$ in $] 0, T]$, we take the $L^{2}(] 0, t[\times \Omega)$-scalar product with $\operatorname{sgn}_{\lambda}(u-v)^{+} \zeta$, the functions $\zeta$ and $\operatorname{sgn}_{\lambda}$ being respectively introduced in the proofs of Theorem 3.1 and Property 3.1. Thus, owing to the monotony of the penalizing operators $\beta(t, x,$.$) and \beta^{*}(t, x,$.$) and to the definition of \operatorname{sgn}_{\lambda}^{+}$, we make sure that for any $t$ of $\left.] 0, T\right]$,

$$
\left.\left\{\beta(t, x, u)-\beta^{*}(t, x, v)\right\} \operatorname{sgn}_{\lambda}(u-v)^{+} \geq 0 \text { a.e. on }\right] 0, t[\times \Omega \text {. }
$$

Accordingly, some integrations by parts and the Lipschitz properties for $\mathbf{f}$ and $g$ provide the next inequality:

$$
\begin{aligned}
\int_{\Omega} I_{\lambda}(u-v)^{+}(t, .) \zeta \mathrm{d} x \leq & \int_{\Omega} I_{\lambda}\left(u_{0}-v_{0}\right)^{+} \mathrm{d} x+M_{g}^{\prime} \int_{Q_{t}}(u-v)^{+} \mathrm{d} x \mathrm{~d} t+A \int_{Q_{t}}(u-v)^{+}\left|\nabla \operatorname{sgn}_{\lambda}(u-v)^{+}\right| \mathrm{d} x \mathrm{~d} t \\
& -\epsilon \int_{Q_{t}} \operatorname{sgn}_{\lambda}(u-v)^{+} \nabla(u-v) \cdot \nabla \zeta \mathrm{d} x \mathrm{~d} t+A \int_{Q_{t}}(u-v)^{+} \operatorname{sgn}_{\lambda}(u-v)^{+}|\nabla \zeta| \mathrm{d} x \mathrm{~d} t .
\end{aligned}
$$

In the right-hand member, for the second line, we use the differential inequality fulfilled by the function $\zeta$ with $\varphi=(u-v)^{+} \operatorname{sgn}_{\lambda}(u-v)^{+}$. Thus,

$$
\begin{aligned}
\int_{\Omega} I_{\lambda}(u-v)^{+}(t, .) \zeta \mathrm{d} x \leq & (A+L \epsilon) \int_{\Sigma_{t}}\left(u_{\Gamma}-v_{\Gamma}\right)^{+} \mathrm{d} \sigma+\int_{\Omega} I_{\lambda}\left(u_{0}-v_{0}\right)^{+} \mathrm{d} x+M_{g}^{\prime} \int_{Q_{t}}(u-v)^{+} \mathrm{d} x \mathrm{~d} t \\
& +A \int_{Q_{t}}(u-v)^{+}\left|\nabla \operatorname{sgn}_{\lambda}(u-v)^{+}\right| \mathrm{d} x \mathrm{~d} t+\epsilon \int_{Q_{t}}(u-v)^{+} \nabla \zeta \cdot \nabla \operatorname{sgn}_{\lambda}(u-v)^{+} \mathrm{d} x \mathrm{~d} t .
\end{aligned}
$$

Due to the Sacks lemma, $\epsilon$ being fixed, each term of the second line goes to 0 when $\lambda$ goes to $0^{+}$. Then, the $\epsilon$-limit and the Gronwall lemma give relation (30).

The consequences of Lemma 4.1 are diverse. Of course, it supplies the monotonistic dependence on boundary data for the solution $u$ to (1),(2),(3) but it also provides some approximation and stability results for $u$ with respect to the associated obstacle functions. Indeed, the next statement holds: 
Property 4.1. Let $\left(\theta_{i, n}\right)_{n>0}, i=1,2$, be two sequences of $W^{1,+\infty}(Q)$-functions such that:

$$
\exists M_{i}>0, \forall n>0,\left\|\theta_{i, n}\right\|_{L^{\infty}(Q)} \leq M_{i} .
$$

For any $n>0$, we denote $v_{n}$ the weak entropy solution to the following bilateral obstacle problem:

$$
\begin{gathered}
\theta_{1, n} \leq v_{n} \leq \theta_{2, n} \text { a.e. in } Q, \\
\left(v_{n}-\theta_{1, n}\right) \mathbb{H}\left(t, x, v_{n}\right) \leq 0,\left(v_{n}-\theta_{2, n}\right) \mathbb{H}\left(t, x, v_{n}\right) \leq 0,\left(v_{n}-\theta_{1, n}\right)\left(v_{n}-\theta_{2, n}\right) \mathbb{H}\left(t, x, v_{n}\right)=0 \text { in } Q, \\
\left.v_{n}=u_{\Gamma} \text { on (a part of }\right) \Sigma, v_{n}(0, .)=u_{0} \text { in } \Omega .
\end{gathered}
$$

That way, if $\left(\theta_{i, n}\right)_{n>0}$ converges toward $\theta_{i}$ in $W^{1,1}(Q)$ as $n$ goes to $+\infty$, then $\left(v_{n}\right)_{n>0}$ strongly converges to $u$ in $L^{r}(Q), 1 \leq r<+\infty$. In addition, if the sequences $\left(\theta_{i, n}\right)_{n>0}$ are similarly monotonistic, then so it is for $\left(v_{n}\right)_{n>n_{0}}$.

Proof. We first observe that on account of the uniform estimate for $\left(\theta_{i, n}\right)_{n>0}$ the sequence $\left(v_{n}\right)_{n>0}$ is bounded in $L^{\infty}(Q)$. So, $n$ being fixed, we refer to the arguments developed in the proof of Theorem 3.1 with $K=K_{n}$ now. Then the convergence hypothesis for the sequences $\left(\theta_{i, n}\right)_{n>0}$ are sufficient to pass to the limit with $n$ in the nonlinear terms $H\left(v_{n}, K_{n}\right), \mathbf{Q}\left(v_{n}, K_{n}\right)$ and $G\left(v_{n}, K_{n}\right)$. The uniqueness of the entropy process solution constructed this way ensures that the whole sequence $\left(v_{n}\right)_{n}$ converges to the weak entropy solution $u$ of obstacle problem (1), (2), (3). Moreover, let us suppose that sequences $\left(\theta_{i, n}\right)_{n>0}$ are non-decreasing. Then, for $n$ large enough $\left(n \geq n_{0}\right)$, for $m>n$,

$$
\theta_{1, n} \leq \theta_{1, m} \leq \theta_{1} \leq \theta_{2, n} \leq \theta_{2, m} \leq \theta_{2} \text { a.e. on } Q .
$$

Due to (30), $v_{n} \leq v_{m} \leq u$ a.e. on $Q$ and the conclusion follows.

We now examine sufficient conditions on data such that obstacle problem (1), (2), (3) is not a free boundary one. Namely, we look for relations between the weak entropy solution $u$ of $(1),(2),(3)$ and $v$, that of the unconstrained problem:

$$
\begin{gathered}
\mathbb{H}(t, x, v)=0 \text { in } Q, \\
v(t, \sigma)=u_{\Gamma} \text { on (a part) of } \Sigma, v(0, .)=u_{0} \text { in } \Omega,
\end{gathered}
$$

and the next statement holds:

\section{Property 4.2 .}

(i) If $\mathbb{H}\left(t, x, \theta_{1}\right) \leq 0$ and $\mathbb{H}\left(t, x, \theta_{2}\right) \geq 0$ a.e. on $Q$, then $u=v$.

(ii) If $v$ belongs to $W^{1,1}(Q) \cap L^{\infty}(Q)$, if $\mathbb{H}\left(t, x, \theta_{1}\right) \geq 0$ and $\mathbb{H}\left(t, x, \theta_{2}\right) \leq 0$ a.e. on $Q$, then:

$$
u=\theta_{1}+\left(v-\theta_{1}\right)^{+}-\left(v-\theta_{2}\right)^{+}
$$

Proof. The point $(i)$ is a consequence of the general result, for any function $\theta$ of $W^{1,+\infty}(Q)$

$$
M_{L}(t) \leq v(t, x)-\theta(t, x) \leq M_{U}(t) \text { a.e. on } Q,
$$

where

$$
M_{L}(t)=-\max \left(\left\|\left(u_{0}-\theta(0, .)\right)^{-}\right\|_{\infty} ;\left\|\left(u_{\Gamma}-\theta_{\left.\right|_{\Gamma}}\right)^{-}\right\|_{\infty}\right) \mathrm{e}^{C_{1}^{*} t}-\frac{\left(e^{C_{1}^{*} t}-1\right)}{C_{1}^{*}}\left\|\mathbb{H}(t, x, \theta)^{+}\right\|_{L^{\infty}(Q)}
$$

and

$$
M_{U}(t)=\max \left(\left\|\left(u_{0}-\theta(0, .)\right)^{+}\right\|_{\infty} ;\left\|\left(u_{\Gamma}-\theta_{\left.\right|_{\Gamma}}\right)^{+}\right\|_{\infty}\right) \mathrm{e}^{C_{1}^{*} t}+\frac{\left(e^{C_{1}^{*} t}-1\right)}{C_{1}^{*}}\left\|\mathbb{H}(t, x, \theta)^{-}\right\|_{L^{\infty}(Q)},
$$


with $C_{1}^{*}$ depending on $\left\|\nabla \theta_{1}\right\|_{\infty}, M_{g}^{\prime}, A$ and on the Lipschitz constants of $\partial_{u} \mathbf{f}$ with respect to its third variable.

Indeed, by using a cut-off method and resorting to the viscous problem the arguments developed in Property 3.1 prove that for any $t$ of $] 0, T]$ (we have dropped index $\epsilon$ ),

$$
\begin{aligned}
\int_{\Omega} I_{\lambda}\left(v(t, .)-M_{L}(t, .)-\theta(t, .)\right)^{-} \mathrm{d} x-\int_{Q_{t}} \mathbb{H}\left(t, x, \theta+M_{L}\right) \operatorname{sgn}_{\lambda}(v- & \left.M_{L}-\theta\right)^{-} \mathrm{d} x \mathrm{~d} t \\
& \quad \leq M_{g}^{\prime} \int_{Q_{t}}\left(v-M_{L}-\theta\right)^{-} \mathrm{d} x \mathrm{~d} t+\epsilon \int_{Q_{t}}|\Delta \theta| \mathrm{d} x \mathrm{~d} t
\end{aligned}
$$

The definition of $M_{L}$ and the Lipschitz conditions for $\mathbf{f}$ and $g$ ensure that $\mathbb{H}\left(t, x, \theta+M_{L}\right)$ is nonpositive a.e. on $Q$. When $\epsilon$ and $\lambda$ tend to $0^{+}$, the minoration for $v$ follows from the Gronwall lemma. The majoration for $v$ is based on the same principles.

Now, by taking account of the obstacle conditions for $u_{0}$ and $u_{\Gamma}$ and the sign conditions for $\mathbb{H}\left(t, x, \theta_{1}\right)$ and $\mathbb{H}\left(t, x, \theta_{2}\right)$ we make sure that $\theta_{1} \leq v \leq \theta_{2}$ a.e. in $Q$. So, to prove that $u=v$ we just have to ensure that $v$ fulfills relations (6), (7) and (8) of Definition 1.1. In fact, the proof of Theorem 3.1 shows that (6), (7), (8) for $v$ are obtained as soon as the viscous solution $v_{\epsilon}$ fulfills (28), which is obviously true.

(ii) Let us denote $w=\theta_{1}+\left(v-\theta_{1}\right)^{+}-\left(v-\theta_{2}\right)^{+}$. By construction, $\theta_{1} \leq w \leq \theta_{2}$ a.e. on $Q$. Therefore, to prove that $w=u$ we have to establish that $w$ fulfills relations (6), (7) and (8). To do so, we first remark that as $k$ is fixed in $[0,1]$ the sign conditions for $\mathbb{H}\left(t, x, \theta_{1}\right)$ and $\mathbb{H}\left(t, x, \theta_{2}\right)$ guarantee that a.e. on $Q$ :

$$
\begin{aligned}
& \left\{\partial_{t} \theta_{1} \operatorname{sgn}\left(v-\theta_{1}\right)^{+}-\partial_{t} \theta_{2} \operatorname{sgn}\left(v-\theta_{2}\right)^{+}-\partial_{t} \theta_{1}\right. \\
& \left.+\{\boldsymbol{\nabla} \cdot \mathbf{f}(t, x, v)+g(t, x, v)\}\left\{\operatorname{sgn}\left(v-\theta_{1}\right)^{+}-\operatorname{sgn}\left(v-\theta_{2}\right)^{+}\right\}\right\} \partial_{1} H(w, K) \\
& \geq\{\boldsymbol{\nabla} \cdot \mathbf{f}(t, x, w)+g(t, x, w)\} \partial_{1} H(w, K)
\end{aligned}
$$

where $K=k\left(\theta_{2}-\theta_{1}\right)+\theta_{1}$ and $(H, \mathbf{Q})$ is a boundary entropy-entropy flux pair (see proof of Th. 3.1). In addition, owing to the regularity of $v,(31)$ is fulfilled a.e. on $Q$. Hence, on the one hand one multiplies with $\operatorname{sgn}\left(v-\theta_{1}\right)^{+} \partial_{1} H(w, K)$ and on the other hand with $-\operatorname{sgn}\left(v-\theta_{2}\right)^{+} \partial_{1} H(w, K)$. By adding up and by considering the previous inequality it ensues:

$$
\left\{\partial_{t} w+\boldsymbol{\nabla} \cdot \mathbf{f}(t, x, w)+g(t, x, w)\right\} \partial_{1} H(w, K) \leq 0 \text { a.e. on } Q \text {. }
$$

As a consequence, as in Theorem 3.1 we prove that $w$ fulfills relation (28) with $\epsilon=0$. So, as mentioned above, one gets $(6),(7),(8)$ for $w$, which completes the proof of Property 4.2.

\section{Conclusion}

To conclude, we have proved the existence and uniqueness of the weak entropy solution for a nonlinear scalar conservation law associated with Dirichlet boundary data and a bilateral obstacle constraint in a bounded domain of $\mathbb{R}^{p}, p \geq 1$. Yet, the numerical approximation this solution remains to be proved. A first study has been achieved in [13] for a quasilinear first-order equation associated with a forced positiveness condition. Through the time-splitting method an $L^{1}$-error bound in $\mathcal{O}(\sqrt{\Delta t})$ is established for the Cauchy problem and only an $L^{1}$-convergence result has been demonstrated for the Dirichlet one so far. 


\section{REFERENCES}

[1] J.M. Ball, A version of the fundamental theorem for young measures, in PDEs and continuum model of phase transition, Lect. Notes Phys. 344, Springer-Verlag, Berlin (1995) 241-259.

[2] C. Bardos, A.Y. LeRoux and J.C. Nedelec, First-order quasilinear equations with boundary conditions. Comm. Partial Differential Equations 4 (1979) 1017-1034.

[3] S. Benharbit, A. Chalabi and J.P. Vila, Numerical viscosity and convergence of finite volume methods for conservation laws with boundary conditions. SIAM J. Numer. Anal. 32 (1995) 775-796.

[4] A. Bensoussan and J.L. Lions, Inéquations variationnelles non linéaires du premier et second ordre. C. R. Acad. Sci. Paris, Sér. A 276 (1973) 1411-1415.

[5] P. Bia and M. Combarnous, Les méthodes thermiques de production des hydrocarbures, Chap. 1 : Transfert de chaleur et de masse. Revue de l'Institut français du pétrole (1975) 359-394.

[6] C. Chainais-Hillairet, Finite volume schemes for a nonlinear hyperbolic equation. Convergence toward the entropy solution and error estimate. ESAIM: M2AN 33 (1999) 129-156.

[7] S. Champier, T. Gallouët and R. Herbin, Convergence of an upstream finite volume scheme for a nonlinear hyperbolic equation on triangular mesh. Numer. Math. 66 (1993) 139-157.

[8] R.J. Diperna, Measure-valued solutions to conservation laws. Arch. Rat. Mech. Anal. 88 (1985) 223-270.

[9] R. Eymard, T. Gallouët and R. Herbin, Existence and uniqueness of the entropy solution to a nonlinear hyperbolic equation. Chin. Ann. Math. 16B (1995) 1-14.

[10] G. Gagneux and M. Madaune-Tort, Analyse mathématique de modèles non linéaires de l'ingénierie pétrolière. Math. Appl. SMAI 22, Springer-Verlag, Berlin (1996).

[11] D. Kröner and M. Rokyta, Convergence of upwing finite volume schemes for scalar conservation laws in two dimensions. SIAM J. Numer. Anal. 31 (1994) 324-343.

[12] S.N. Kruskov, First-order quasilinear equations in several independent variables. Math. USSR Sb. 10 (1970) $217-243$.

[13] L. Lévi and F. Peyroutet, A time-fractional step method for conservation law related obstacle problem. (Preprint 99/37. Laboratory of Applied Math., ERS 2055, Pau University.), Adv. Appl. Math. (to appear).

[14] F. Otto, Conservation laws in bounded domains, uniqueness and existence via parabolic approximation, in Weak and measurevalued solutions to evolutionary PDE's, J. Malek, J. Necas, M. Rokyta and M. Ruzicka Eds., Chapman \& Hall, London (1996) 95-143.

[15] A. Szepessy, Measure solutions to scalar conservation laws with boundary conditions. Arch. Rat. Mech. Anal. 107 (1989) $181-193$.

[16] A. Szepessy, Convergence of a streamline diffusion finite element method for scalar conservation laws with boundary condition. ESAIM: M2AN 25 (1991) 749-782.

[17] L. Tartar, Compensated compactness and applications to partial differential equations, in Nonlinear analysis and mechanics. Heriot-Watt Symposium, R.J. Knops Ed., Res. Notes Math. 4, Pitman Press, New-York (1979).

[18] G. Vallet, Dirichlet problem for nonlinear conservation law. Revista Matematica Complutense XIII (2000) 1-20.

[19] M.H. Vignal, Convergence of a finite volume scheme for elliptic-hyperbolic system. RAIRO: Modél. Math. Anal. Numér. 30 (1996) 841-872.

To access this journal online:

www.edpsciences.org 